\title{
Raum für Veränderung: eine raumsoziologische Perspektive auf internationale Verhandlungen, institutionelle Interaktion und Normwandel
}

\section{Linda Wallbott}

\section{Einleitung}

Dieser Beitrag wirft eine theoretisch-konzeptionelle Perspektive auf die Wechselwirkungen zwischen internationalen Institutionen und ihre Relevanz für Normwandel in der globalen Politik. ${ }^{1}$ Jene ist wesentlich gekennzeichnet durch die Unterteilung in sektorale und distinkte institutionelle Regelungsarrangements. Spezialisierte Regime begünstigen einerseits die zielgerichtete Problembearbeitung und Herausbildung themenspezifischer Expertise. Andererseits entstehen latente, aber auch manifeste Konflikte (Zelli 2008), wenn zwei Ordnungsmodelle aufeinanderprallen, d.h. wenn funktional verbundene Institutionen (Young 1999; Stokke 2001; Gehring/ Oberthür 2009), die sich auf denselben Regelungsgegenstand bzw. Zielkontext beziehen, hinsichtlich ihrer zentralen formalen Normen und Akteurskonstellationen variieren (für Strategien des Umgangs mit Konflikten zwischen verschiedenen Ordnungen siehe Zimmermann et al. 2013). Bisher fokussierte sich die Forschung zu Wechselwirkungen internationaler Institutionen auf die negativen Folgen einer fragmentierten Global Governance-Architektur ${ }^{2}$ für die Effektivität vertikaler Politik-Implementierung. Insbesondere die Untersuchung formaler und funktionaler Aspekte stand hierbei im Zentrum der Aufmerksamkeit.

Aber auch internationale Verhandlungen sind bereits durch das Strukturmerkmal institutioneller Fragmentierung beeinflusst. So ist globale Politik weit davon entfernt, ein kohärentes Ordnungssystem mit für alle AkteurInnen gleichermaßen berechenbaren Räumen der Politikgestaltung zu sein. So wurde bisher vor allem betont, dass ressourcenstarke staatliche Ak-

1 Ich danke Nicole Deitelhoff, Martina Löw, Carmen Wunderlich, Stefanie Herr, Ben Kamis, Thorsten Thiel, Jannik Pfister und den HerausgeberInnen für wertvolle Kommentare zu einer früheren Version dieses Aufsatzes.

2 Dem Architekturbegriff wird im Gegensatz zum Begriff der globalen Ordnung eine relativ größere Wertneutralität zugesprochen (Biermann et al. 2010: 16). 
teurInnen aufgrund ihrer materiell und kognitiv gestützten, verhältnismäßig größeren Mobilität in der Lage sind, von der Fragmentierung bzw. polyzentrischen Struktur (Ostrom 2010) des globalen politischen Systems zu profitieren und institutionelle Strukturen zu ihrem Vorteil zu bearbeiten. Mittels forum shopping bringen sie sich gezielt in denjenigen institutionellen Foren ein, von denen sie sich den größten Nutzen versprechen. Im Gegensatz dazu sind beispielsweise Entwicklungsländer und viele nicht-staatliche AkteurInnen häufig nicht in der Lage, Delegationen mit ähnlicher Personenstärke und materieller Ausstattung wie Industrieländer und wirtschaftliche Interessenorganisationen in internationale Verhandlungen zu entsenden. Angesichts der parallelen Bearbeitung thematisch ähnlicher Sachfragen in unterschiedlichen Institutionen, der Gleichzeitigkeit von Arbeitsgruppentreffen innerhalb einzelner Verhandlungen, der Relevanz von Ausgleichszahlungen und Paketlösungen aber auch der formalen Kriterien internationaler Organisationen (Mandat, Mitgliedschaft, Stimmberechtigung, Verfahrensregeln) wird daher (nicht-)staatlichen AkteurInnen, die weniger stark mit Ressourcen ausgestattet sind und in dieser Hinsicht als schwach bezeichnet werden können, lediglich eine eingeschränkte agency, also Handlungs- und Problemlösungsfähigkeit in internationalen Verhandlungen und mit Blick auf Kapazitäten zum Management institutioneller Wechselwirkung, zugesprochen. ${ }^{3}$ Allerdings wird in diesem Beitrag ein konzeptioneller Rahmen zur Analyse einer fragmentierten internationalen Institutionen-Landschaft entwickelt, der argumentiert, dass auch nicht-hegemoniale AkteurInnen intentional und zielgerichtet zur Konstruktion und Gestaltung von Räumen institutioneller Wechselwirkung beitragen und dass diese neuen Räume dann jenseits von Formalität und Funktionalität auch als überlappende argumentative bzw. diskursive Felder in internationalen Verhandlungen wirksam werden. ${ }^{4}$ In diesem Prozess können Normen gleichzeitig mit neuen Bedeutungen belegt werden. So initiieren NormunternehmerInnen institutionelle Wechselwirkungen absichtsvoll und nutzen sie, um in internationalen Verhandlungen das De-

3 In der Verwendung des agency-Begriffs folge ich hier Biermann et al. (2009) und Schroeder (2010) in der Hinsicht, dass ein(e) AgentIn ein(e) autoritative(r) AkteurIn ist, der oder die über Legitimität und Kapazität zur Ausübung von Macht verfügt. Für Fragestellungen zum Verhältnis von Global Governance-Architektur und agency vgl. auch Biermann et al. (2009: 39).

4 Gleichwohl sind diejenigen AkteurInnen, die in internationalen Verhandlungen vertreten sind und durch Interdependenz-Management die Durchsetzung ihrer Interessen unterstützen können, stets schon Teil einer transnationalen Elite. 
sign internationaler Institutionen zu beeinflussen und dominante Ordnungsvorstellungen herauszufordern (Wallbott 2019).

Indem dieser Beitrag also die bis dato nur unzureichend berücksichtigte und ausgearbeitete agency-Komponente bei der Neugestaltung von Räumlichkeit und der Verschiebung institutioneller wie normativer Grenzen betont, ergänzt er die noch junge Forschung zum Management institutioneller Interdependenz. Darüber hinaus zielt der Beitrag auf die bestehende institutionalistische Lücke der Normenforschung, die bisher den analytischen Schwerpunkt vor allem auf die Untersuchung der Beiträge unterschiedlicher Akteursgruppen legt (Coleman 2013). Insgesamt wird durch die In-Beziehung-Setzung von Diskurs-bezogener agency und dynamischer Normenqualität einerseits und Management institutioneller Interdependenz andererseits sowohl die Literatur zu Normen- wie auch zur Institutionenforschung bereichert. Diese konzeptionelle Verbindung wird durch eine prozessuale und relationale raumsoziologische Perspektive ermöglicht bzw. gestützt, die gleichberechtigt die Ordnungs- und Handlungsdimension von Raum anerkennt (Löw 2001). Raum ist demnach nicht nur territorial oder geopolitisch zu verstehen, sondern auch als soziales Konstrukt, das jenseits einer ausschließlich formalistischen oder materialistischen Wirksamkeit dynamisch ist. Durch diesen Blickwinkel, der eine kognitive Konstruktionsleistung von AkteurInnen für die Konstitution von Raum voraussetzt, ist es möglich, die Entstehung institutioneller Wechselwirkung mit diskursivem Handeln von NormunternehmerInnen zu verbinden.

Im Folgenden wird zunächst die Forschungslücke an der Intersektion von Arbeiten zu Normwandel und NormunternehmerInnentum auf der einen und institutioneller Interaktion auf der anderen Seite skizziert (Abschnitt 2). Auf dieser Basis wird ein alternatives, raumsoziologisches Konzept zum Management von Institutionen und institutioneller Interdependenz erstellt, dessen definitorisches Element in der Verbindung von diskursiver agency und emergenter räumlicher Struktur liegt. Dieser konzeptionelle Ansatz wird dann auf die Vereinten Nationen und internationale Verhandlungen bezogen (Abschnitt 3). Abschließend werden weiterführende kausalanalytische und normative Untersuchungsperspektiven für Institutionen- und Normenforschung aufgezeigt, die sich aus diesem konzeptionellen Beitrag ableiten lassen (Abschnitt 4). 


\section{Von Regime-Analyse zu einem Forschungsprogramm institutioneller Interaktion}

\subsection{Zum Charakter internationaler Politikgestaltung}

Vor dem Hintergrund der fragmentierten globalen Rechtsordnung war das vorherrschende Verständnis der Internationalen Beziehungen lange Zeit, dass sich AkteurInnen in themenspezifischen Institutionen ${ }^{5}$ unabhängig voneinander mit klar abgrenzbaren, eigenständigen Problemen auseinandersetzen (Victor et al. 1998; Miles et al. 2002). Im Rahmen der polyzentrischen (Ostrom 2010) oder auch heterarchischen (Daase/Deitelhoff 2014) Ordnungsstruktur des internationalen Systems sind die Beziehungen zwischen den einzelnen Teilordnungen mit ihren eigenen Mandaten und Rationalitäten nicht durch eine zentrale Autorität gesteuert. Vor diesem Hintergrund standen bei der Analyse internationaler Regime lange Zeit die funktionalen Bedingungen ihrer jeweiligen Entstehung, Effektivität und ihres institutionellen Designs - Gestaltung der formalen und informellen Prozeduren, Praktiken, Regeln und Normen, durch welche die Arbeitsvorgänge von Institutionen bestimmt und die beteiligten AkteurInnen in Relation zueinander gesetzt werden - im Mittelpunkt (Haas et al. 1993; Young 1999; Miles et al. 2002; Breitmeier et al. 2011). Hierbei dominierten in der Regel rationalistische Perspektiven (Gruber 2000; Koremenos et al. 2004; Pollack 2003). In der vergangenen Dekade schließlich richtete sich das Interesse zunehmend auf die Untersuchung der funktionalen Verbindungen zwischen verschiedenen Sektoren und Regimen, die sich mit divergierenden Handlungsaufforderungen auf denselben Zielkontext beziehen (Rosendal 2001a, 2001b; Stokke 2001). Dabei werden zunehmend auch die Kausalmechanismen der wechselseitigen Beeinflussung internationaler Institutionen in den Blick genommen, die auch auf Normwandel-Prozesse bezogen werden können. ${ }^{6}$

5 Institutionen umfassen »clusters of rights, rules, and decision-making procedures that give rise to social practices, assign roles to participants in these practices, and govern interactions among occupants of these roles" (Schroeder 2008: 51). In einem engeren Verständnis sind Institutionen Organisationen, d.h. bezogen auf die internationale Ebene derartige strukturelle Arrangements, die einen normativen Rahmen, eine spezifische Mitgliedschaft von Staaten (und gegebenenfalls nicht-staatlichen AkteurInnen) und einen bürokratischen (Verwaltungs-)Apparat verbinden und prinzipiell effektive Regeln formulieren können (Biermann/Siebenhüner et al. 2009: 39).

6 Institutionelle Wechselbeziehungen wurden bisher vor allem in der Vergleichenden Politikwissenschaft und in der Forschung zu Transfer und Diffusion von Nor- 


\subsection{Mechanismen institutioneller Interaktion}

Grundsätzlich kann die Entwicklung und Umsetzung des Regelwerkes einer Institution durch benachbarte Institutionen beeinflusst werden, indem Handlungsoptionen entweder begrenzt oder eröffnet werden (Gehring 2007). Bedingung hierfür ist der kausale Zusammenhang der beteiligten Institutionen, ohne den lediglich eine parallele, aber unverbundene Entwicklung besteht (Gehring 2007: 94; Stokke/Oberthür 2011b: 4; vgl. die Unterscheidung zwischen engem und weitem Diffusionsbegriff in Holzinger et al. 2007: 14). Auf der Basis früher effektivitätsorientierter Taxonomien (Young 1996, 2002; Stokke 2000) wurde schließlich ein vierteiliges idealtypisches Set möglicher Kausalmechanismen institutioneller Wechselwirkung entwickelt (Gehring/Oberthür 2009, 2011). Die kognitive Interaktion (cognitive interaction) basiert demnach auf Einsicht und Überzeugung und wird durch die Verbreitung von Wissen vorangetrieben, wenn die Zielorganisation sich am Modell der Ursprungsorganisation orientiert. Die verpflichtungsbasierte Interaktion (interaction through commitment) tritt dann auf, wenn Organisationen über geteilte Mitgliedschaft verfügen und sich im gleichen Politikfeld bewegen. Dann können beispielsweise formale Verpflichtungen zwischen ihnen übertragen werden oder ein zusätzliches Politikinstrument wird von beiden Institutionen geschaffen. Im Falle von verhaltensbasierter Interaktion (behavioural interaction) beeinflussen die Maßnahmen einer Institution als positive oder negative Externalitäten die Implementierung der Vorgaben einer anderen. Schlussendlich gründet die impact-basierte Interaktion (impact level interaction) auf der funktionalen Verbindung der Steuerungssysteme und manifestiert sich während der Umsetzung unterschiedlicher Politikvorgaben im Zielkontext, also vor Ort.

Für Normwandel in internationalen Verhandlungen scheinen vor allem die ersten beiden Kausalmechanismen relevant, die sich auf institutionellen output (z.B. Resolutionen) beziehen. In jedem Fall allerdings müssen Forderungen nach normativer Veränderung von AgentInnen formuliert und in den politischen Aushandlungsprozess eingespeist werden. Bisher allerdings wurden die Möglichkeiten von Wechselwirkung zwischen internationalen Institutionen recht formalistisch und statisch unter Rückgriff auf die jeweils geltenden Mandate der Organisationen und abweichende Mitgliedschaften bewertet. So suggeriert auch die begriffliche Unterschei-

men behandelt. Diese Arbeiten untersuchen vor allem die Konvergenz nationaler

Institutionen, Strukturen und Politiken (Holzinger et al. 2007). 
dung in Ursprungs- und Zielorganisation eine unidirektionale kausale Wirkungsrichtung, wodurch wiederum breitere Dynamiken ausgeblendet werden.

\subsection{Management institutioneller Interdependenz}

In Arbeiten zu institutioneller Wechselwirkung wird denn auch zunehmend das Fehlen einer prozessualen oder diskursanalytischen und normativ gehaltvolleren Perspektive bemängelt (Zelli et al. 2012; Gehring/Oberthür 2011: 47). Einen Einstiegspunkt in eine solche Untersuchung bietet die jüngst aufgekommene Rede vom Management institutioneller Interdependenz (interplay management) (Oberthür 2009; Oberthür/Stokke 2011a). Diese impliziert, dass AkteurInnen in der Lage sind, mit potentieller institutioneller Interaktion in einer antizipierenden, reflexiven, intentionalen, koordinierten und zielorientierten Weise umzugehen. Das ManagementKonzept unterscheidet sich von Begriffen wie Verbindung, Wechselwirkung oder spontane Reaktion durch seine absichtsvolle und vorausschauende Komponente (Gehring/Oberthür 2009; Oberthür/Stokke 2011b: 6-10). So betont die Management-Perspektive, dass institutionelle Strukturen gezielt für konkrete Steuerungsziele aufgebaut bzw. genutzt werden können (vgl. Busch/Jörgens 2007: 78; Oberthür/Gehring 2011: 44).

Die zielgerichtete Bearbeitung politischer Wechselwirkung zwischen Institutionen wurde bisher aber primär mit Blick auf die Minimierung von Konflikten betrachtet. Eine systematische und grundlegende Untersuchung der agency-Komponente im Kontext des Managements institutioneller Interdependenz hingegen fehlt (bereits Selin/VanDeever 2003; Oberthür/Gehring 2011: 44; Oberthür/Stokke 2011c: 335-336; grundlegend Biermann et al. 2009: 36-43). Wenn die Bedeutung institutioneller Komplexität und Interaktion für die Positionierung von AkteurInnen in internationalen Verhandlungen bisher in der Literatur berücksichtigt wurde, dann vor allem durch die Annahme, dass sie mächtigen Staaten nutzen. Mittels forum shopping verlagern diese Debatten in solche Institutionen, in denen sie unerwünschten formalen Vorgaben beispielsweise durch Ausgleichszahlungen am ehesten aus dem Weg gehen können, oder in denen eine möglichst große Staatengruppe von den Folgewirkungen der für sie günstigen Regulierung betroffen sein wird (bereits Krasner 1983: 16; Drezner 2009; Braithwaite/Drahos 2000; Benvenisti/Downs 2007; Busch 2007).

Raumsoziologisch gesprochen resultieren diese analytischen Verengungen aus der ontologischen Trennung von institutionellem/organisationalem Raum und sozialer/politischer Praxis. Auf dieser absolutistischen 
Grundannahme aufbauend wird nicht die Konstitution von Raum selbst untersucht, sondern lediglich das Handeln innerhalb bestehender und als gegeben konzipierter Strukturen. ${ }^{7}$ Institutionelle Räume werden hier als Container oder Behälter konzeptualisiert. So wird »ein Raum unterstellt, der das Handeln aller gleichermaßen vorstrukturiert " (Löw 2001: 64) und innerhalb dessen zwar Bewegungen stattfinden können, der aber selbst nicht bewegt wird, der also nicht dynamisch ist (Löw 2001: 65). ${ }^{8}$ Die Relevanz ideeller und symbolischer Verknüpfung (Löw 2001: 64) für Raumgestaltung kann in einem solchen Vorgehen nicht berücksichtigt werden ebenso wenig wie die Veränderung des Bedeutungsgehalts von Normen. So geraten insbesondere solche normunternehmerischen Aktivitäten aus dem Blickfeld, die sich über organisationale Grenzen hinweg erstrecken und durch den gezielten Aufbau argumentativer und symbolischer Räume Normwandel und institutionelles (Neu-)Design vorantreiben (wollen). Die absichtsvolle und zielgerichtete Gestaltung räumlicher Strukturen durch nicht-hegemoniale Staaten ${ }^{9}$ und verschiedene nicht-staatliche AkteurInnen wurde in den entsprechenden Arbeiten bisher meist vernachlässigt (eine Ausnahme ist Helfer 2004). Daher fanden solche strategischen Aktivitäten von NormunternehmerInnen bisher kaum Beachtung, die auf diskursiven und funktionalen Räumen aufbauen, die sich durch die Überlappung von

7 Eine prominente Position wird von Ernesto Laclau vertreten, der Raum als Bereich von Determination und Stillstand versteht. Er geht von einem geschlossenen und selbstreferentiellen - damit zyklischen, aber auch kohärenten - System aus, das stets nur durch interne Ursachen modifiziert wird, seine eigenen Begriffe aber nicht grundsätzlich umdeuten kann. In diesem Verständnis ist Räumlichkeit »die Koexistenz in einer Struktur, welche die Positivität all ihrer Begriffe begründet« (Laclau 1990: 69). Dynamik im Sinne einer grundlegenden Veränderung kann demnach nur als zeitliche Dislokation auftreten, "welche die festgelegten Begriffe des Systems unterbricht. Weil dem Räumlichen die Dislokation fehlt, ist in ihm keine Möglichkeit der Politik enthalten« (Massey 2008 [1992]: 114). Durch den Raum wird, Laclau folgend, nur repräsentiert - wodurch wiederum politische Prozesse abgeschlossen werden. Unterschiedliche Positionen können demnach lediglich in verschiedenen, letzten Endes voneinander abgetrennten Räumen repräsentiert werden (Massey 2008 [1992]: 115). So ist für ihn das entgrenzte Reale letzten Endes zeitlich, der Raum lediglich "mythische[r] Natur« (Laclau 1990: 68) und entpolitisiert.

8 Das Gleiche gilt für verhaltens- und impact-basierte Interaktion: Das hier zugrunde liegende Raumverständnis ist primär ein absolutistisches und ortsbezogenes bzw. territoriales, da angenommen wird, dass Raum an sich existiert und der Bezugspunkt für Handlungen ist (vgl. Löw 2001: 63-65).

9 Eine weitere Lücke theoriegeleiteter empirischer Arbeiten zu NormunternehmerInnen bezieht sich darauf, auch nicht-hegemoniale staatliche AkteurInnen als solche anzuerkennen (aber vgl. Wunderlich 2013; Wallbott 2014a). 
Institutionen ergeben bzw. die von diesen AkteurInnen gezielt hergestellt werden, um ihre Handlungskapazitäten zu erweitern und die Gestaltung einer politischen Ordnungsstruktur jenseits des Nationalstaats aktiv zu beeinflussen.

Zwar werden framing und die spezifische Darstellung von Wissensbeständen über institutionelle Grenzen hinweg in Anlehnung an die Kausalmechanismen kognitive und verpflichtungsbasierte Interaktion in der Tat als wesentliche Faktoren von Interdependenz-Management benannt (Oberthür/Stokke 2011c: 321). Ebenso merken AutorInnen an, dass Ausprägungen und Effekte institutioneller Interaktion unter Berücksichtigung der normativen Grundlagen der beteiligten Institutionen zu untersuchen seien (Oberthür/Gehring 2011: 33; Zelli et al. 2012). Allerdings wurden inter-institutionelle Räume in der Literatur bisher nicht als Felder von Auseinandersetzungen über solche Normen, über Regulierungsinhalte, Partizipation und Deutungshoheit - in anderen Worten: als Felder von Kontestation - konzipiert, die das Design internationaler Institutionen beeinflussen würden. Es mangelt der Forschung zur Gestaltung institutioneller Räume und institutioneller Interaktion also bis dato an solchen Arbeiten, die sich jenseits einer Analyse ihrer Implikationen für unmittelbare Problemlösung dezidiert mit den normativen Dynamiken von politischer Transnationalisierung (Zürn et al. 2007) auseinandersetzen und dies in einen größeren theoretischen Zusammenhang mit Arbeiten zu Normwandel, NormunternehmerInnen und horizontalen Dynamiken zwischen internationalen Institutionen stellen. Hierbei bietet insbesondere die Erfassung von entsprechenden Interaktions-Prozessen und Bedeutungszuschreibungen während der Verhandlungsphase internationaler Politik eine neue Perspektive.

\subsection{NormunternehmerInnen als ManagerInnen institutioneller Interdependenz}

So lohnt in diesem Zusammenhang ein frischer Blick auf transnationale NormunternehmerInnen (Finnemore/Sikkink 1998), die anerkanntermaßen eine maßgebliche Rolle für die Verbreitung von Normen spielen. $\mathrm{Zu}$ diesem Zweck nehmen sie Bezug auf etablierte Paradigmen und normative Strukturen internationaler Politik (exemplarisch Holzscheiter 2005), verständigen sich über Verhandlungsgegenstände außerhalb der eigentlichen Verhandlungsarena (Deitelhoff 2006) und nutzen eine institutionalisierte Plattform, um ihre alternativen Ordnungsvorstellungen zu verbreiten (Finnemore/Sikkink 1998: 899). Dabei wurden NormunternehmerInnen bisher vor allem als "norm transmitters « konzeptualisiert, ohne dabei 
die interaktive Dimension von Normdynamiken zu reflektieren (Wunderlich 2013: 24-25). Ihre Handlungen wurden in der Regel in Bezug auf einen bestimmten, gegebenen Raum analysiert; weniger wurde gefragt, wie sich durch die von ihnen betriebene Anordnung und Verknüpfung sozialer Güter und AkteurInnen relationale Räume zwischen internationalen Institutionen überhaupt erst konstituieren. ${ }^{10}$ Dabei sind NormunternehmerInnen nicht nur Brückenbauer zwischen verschiedenen institutionellen und geographischen Räumen, sondern definieren hierdurch auch neue Standards und Grenzen. So kann sich durch kognitive und verpflichtungsbasierte Interaktion der Geltungsbereich von Normen in geographischer (Gebiet), personeller (AkteurInnen), sachlicher (Fachthemen) oder zeitlicher (Dauer) Hinsicht ändern. Die Neuziehung normativer Grenzen zeigt sich dabei durch Erweiterung oder Einschränkung des jeweiligen Geltungsbereichs und kann je nach Tiefe und Reichweite der tatsächlichen Veränderung als revolutionär oder reformistisch eingeschätzt werden (Wallbott 2014b).

Vor diesem Hintergrund wird in diesem Beitrag vorgeschlagen, die Relevanz von Raum nicht nur auf die "choice of arenas" (Young 2002: 118) zu beziehen. Vielmehr zeigt sie sich für NormunternehmerInnen in der gezielten diskursiven Verknüpfung unterschiedlicher organisationaler Räume, wodurch ein dynamisches inter-institutionelles Feld geschaffen wird (Wallbott 2014b; 2019). Die Konstitution diskursiver Räumlichkeit durch NormunternehmerInnen mittels gezielter Mobilisierung von Wissen (cognitive interaction) und argumentativen Ressourcen (interaction through commitment/normative interplay) aus unterschiedlichen institutionellen Kontexten impliziert dabei sowohl die relationale (An-)Ordnung von sozialen Gütern und AkteurInnen wie auch ihre Verknüpfung und Sinnstiftung. Da Räume sich aber eben auch immer über das definieren, was ihnen äußerlich ist, soll es, in anderen Worten, also darum gehen, Prozesse der Neugestaltung und Aushandlung von institutionellen, normativen und sozialen Grenzen zu erfassen.

10 Katharina Coleman geht soweit, der Normenforschung generell abzusprechen, die Relevanz institutioneller Räume für Normgestaltung und Normsetzung - zugunsten eines starken Fokus auf agency - adäquat zu berücksichtigen (Coleman 2013: 164). 


\section{Veränderung im (inter-)institutionellen Raum: Handeln in Strukturen und strukturbildendes Handeln in internationalen Verhandlungen}

\subsection{Eine relationale raumsoziologische Perspektive auf institutionelle Interaktion}

In diesem Beitrag wird also argumentiert, dass die Beantwortung der Frage, ob und vor allem wie sich die themenspezifischen Teilordnungen des internationalen Systems wechselseitig beeinflussen erst dann möglich ist, wenn ein prozessuales Raumverständnis zu Grunde gelegt wird, in dem diskursive, institutionelle und normative Dynamiken aber auch machttheoretische Erwägungen zu Normwandel zusammengefügt werden. ${ }^{11}$ So lautet die zentrale Arbeitshypothese, die aus diesem konzeptionellen Beitrag abgeleitet werden soll, dass AkteurInnen ihre Autorität, Legitimität und Kapazität als NormunternehmerInnen und AgentInnen durch die zielgerichtete diskursive Gestaltung von Räumen institutioneller Interaktion steigern und hierdurch Normwandel initiieren (können). Insofern kann das Management institutioneller Interdependenz nicht nur - wie bisher - als Ausdruck, sondern komplementär auch als instrumentelles Vehikel zur

11 Durch den breiten konzeptionellen Fokus, der sowohl die diskursiven/kontestativen, institutionellen wie auch die normativen Dynamiken der AkteurIn-StrukturBeziehungen umfasst, unterscheidet sich diese Perspektive von verwandten Ansätzen wie dem »Reflexiven Institutionalismus" (Keohane 1989) und dem »Akteurszentrierten Institutionalismus« (Mayntz/Scharpf 1995; Scharpf 2000), die noch einem konventionellen und vergleichsweise statischen Verständnis von Normen verhaftet bleiben (Schmidt 2008: 304; Wiener/Puetter 2009).

Der »Diskursive Institutionalismus « (Schmidt 2008, 2010) ist dem hier vorgestellten Ansatz insofern ähnlich, als das Wechselspiel zwischen Diskursen und institutioneller Entwicklung hauptsächlicher Untersuchungsgegenstand sein soll. Gleichwohl können durch eine raumsoziologisch-diskurstheoretische Perspektive auf institutionelle Interaktion wesentliche Kritikpunkte umgangen werden, die immer wieder gegen Schmidt vorgebracht werden. So argumentiert beispielsweise Bell (2011: 844), dass eine Überbetonung der Rolle von Sprache und Diskurs Gefahr laufe, die Gewinne institutionalistischer Forschung - nämlich die Bildung eines Gegengewichts zum Behaviorismus - zu verspielen. Ähnlich kritisieren Freistein/Liste (2011: 11), dass Schmidt entgegen ihres eigenen Anspruchs Diskurs und Institution letzten Endes einander doch dialektisch gegenüberstelle, dabei aber die strukturierende Funktion institutioneller Praktiken (beispielsweise für die Festlegung von SprecherInpositionen), die für AkteurInnen nicht immer zugänglich seien, nicht berücksichtige. Im Gegensatz dazu erkennt die hier gewählte und ausgeführte Perspektive die Dualität von Raum in der Hinsicht an, dass (räumliche) Strukturen zwar einerseits »im Handeln verwirklicht werden« (Löw 2001: 172), dass sie ihrerseits das Handeln aber auch strukturieren. 
Genese von produktiver Macht (Barnett/Duvall 2005) betrachtet werden, wie im Folgenden erörtert wird.

Sowohl institutionelles (Re-)Design, also die (Neu-)Formulierung von allgemein verbindlichen Regeln, wie auch normunternehmerische Aktivitäten können dabei raumsoziologisch beschrieben werden als solche »Bestrebungen, die [...] AkteurInnen für eine inszenierte Raumbildung ergreifen. Sie verfolgen damit die Absicht, eine funktional unzureichende räumliche Organisation zu verbessern oder sie nach eigenen Interessensgesichtspunkten umzuändern « (Bürk 2006: 105; Keim 2003). Diese Organisation des Nebeneinanders, der Verteilungsstrukturen und der Platzierungen von sozialen Gütern ${ }^{12}$ und Handelnden wird auch als spacing bezeichnet. Spacing ist stets durch Aushandlungs- und Verhandlungsprozesse bestimmt (Löw 2001: 225, 228) und kann gleichermaßen Strategie wie auch Ziel politischer AkteurInnen sein. Diese Anordnung ist dabei auch immer Grenzziehung (Weidenhaus 2015), also die Differenzierung zwischen einem Innen und Außen bezogen auf geographische, soziale und normative Kategorien. Allerdings führt eine solche Ordnungsleistung erst dann zur Konstituierung eines Raums, wenn Güter und Personen über Wahrnehmungs-, Vorstellungs- oder Erinnerungsprozesse zueinander in Beziehung gesetzt werden (Löw 2001: 159). Diese primär symbolische Verknüpfung und Bedeutungszuschreibung wird auch als Syntheseleistung bezeichnet, die durch einmalige und wiederholende Sprachäußerungen nach außen getragen werden kann. So ist »bei den Räumen des Politischen an diejenigen Räume zu denken, die durch bestimmte Semantiken immer wieder konstruiert werden" (Schroer 2006: 186). Spacing und Syntheseleistung sind somit die zwei grundsätzlich unterschiedlichen, aber notwendigen und gleichzeitig auftretenden Prozesse von Raumkonstitution (Löw 2001: 158-161). Ein Raum erbringt somit stets eine strukturierende Ordnungsleistung, impliziert aber auch die Handlung des Anordnens und der Gestaltung. Eine raumsoziologische Analyse von internationalen Verhandlungen und Normwandel bedeutet demnach, dass die Organisation der institutionellen Wechselbeziehungen sowohl unter »Berücksichtigung der sozialen Konstruiertheit als auch der Materialisierung« vorgenommen werden muss (Löw 2001: 66).

12 Soziale Güter haben sowohl eine materielle (physische) wie auch eine symbolische Dimension. Alle Körper entfalten außerdem eine sinnliche Außenwirkung, schaffen Atmosphäre durch Mimik, Gestik und Sprache, aber auch durch Geräusche und Gerüche. 
Kritische Raumforschung zeichnet sich hierbei dadurch aus, dass sie sich eben nicht für den Raum an sich interessiert, sondern primär dafür, wie Raum konstruiert wird und welche Rolle er in sozialen Prozessen und für Kritik und Wandel spielt (Lefebvre 2006 [1974]; Harvey 1973). Sie erfordert also die Untersuchung der Prozesse, durch die Güter und AkteurInnen angeordnet und in spezifischer Weise miteinander verknüpft werden. Eine solche Perspektive verschiebt das Analyseinteresse hinsichtlich institutioneller Interaktion und Normwandel auf (1) die Bedingungen und Effekte von Raum-Produktion, (2) und auf die Frage, welche AkteurInnen (bzw. Interessen oder Positionen) in welchen Räumen wie repräsentiert sind und (3) wie sie diese im Umkehrschluss durch aktives und zielgerichtetes Handeln (Management) selbst konstituieren. Hier wird angenommen, dass Raumkonstitution ein Prozess der Produktion aber auch des Konfliktaustrags um soziale und politische Ordnungen ist, um den Verlauf, die Durchlässigkeit und die Bedeutung von Grenzen. Die Annahme, dass auch nicht-hegemoniale AkteurInnen räumliche Strukturen zwischen internationalen Institutionen nutzen bzw. aktiv gestalten, um sich - z.B. durch den Transfer von Wissen und Expertise - in internationalen Verhandlungen Gehör zu verschaffen und somit potentiell zum (Neu-)Design internationaler Institutionen beitragen, kulminiert als konzeptionelle Verbindung von agency und Struktur somit in einem kritisch-relationalen Raumbegriff, der die Umstrittenheit der Raumbildung anerkennt. Dieser Raum ist gleichermaßen sozial konstruierte und sozial relevante historischinstitutionelle Sphäre und interaktive ideelle Dimension: er ist das Produkt konkreter sozialer Praktiken. ${ }^{13}$

Der Raum internationaler Institutionen bildet sich also stets sowohl strukturalistisch-formal, aber auch durch ideelle und differentielle Relationen von AkteurInnen ab (vgl. auch Günzel 2008: 222). ${ }^{14}$ Insofern scheint es angemessen, die Priorisierung strukturalistisch-formaler Bindungen zwischen internationalen Teilordnungen zugunsten einer Berücksichtigung

13 Aber eben nicht im deterministisch empiristischen Sinne wie ein Großteil der Literatur, die sich im Anschluss an die einschlägige Vorarbeit von Soja (1989) dem spatial turn zurechnet, sich aber mit realräumlichen Orten befasst und damit letzten Endes auf den geometrischen Ursprung von Raumdebatten zurückläuft.

14 Ein ähnliches Gegensatzpaar entwickelten De Certeau (2006 [1980]) und Merleau-Ponty (2003 [1961]); zum Konnex von Organisation und Raum aus systemtheoretischer Perspektive vgl. Drepper (2003). 
übergreifender sozial-politischer Felder ${ }^{15}$ aufzugeben. Inter-institutionelle Räume werden damit nicht mehr als etwas Äußeres gegenüber sozialen/ politischen Prozessen oder als Bereiche der Stagnation begriffen, sondern als Orte der Akkumulation und (Re-)Produktion materieller, ideeller und symbolischer Ressourcen und Bedeutungen. ${ }^{16}$ Dies bedeutet für die Analyse institutioneller Wechselwirkung, dass eine Beeinflussung von Teilordnungen des heterarchischen Ordnungssystems auch jenseits der formalen und nach Effizienzkriterien beurteilten Übertragung von Normen denkbar scheint. Die Ausgangsvermutung ist, dass die Interessen und Identitäten von AkteurInnen in einer Teilordnung auch von der Positionierung, von den Regeln und Argumenten in anderen Teilordnungen beeinflusst sind. Voraussetzung für die Verknüpfung dieser Teilordnungen und für die Übertragung materieller und ideeller Ressourcen zwischen ihnen ist gleichwohl eine aktive agency, die darauf baut, dass sich die beteiligten AkteurInnen der Möglichkeit der Verknüpfung unterschiedlicher Teilordnungen bewusst sind (vgl. auch Holzinger et al. 2007: 16). ${ }^{17}$ Besonders solche AkteurInnen, die sich in Personalunion in unterschiedlichen Funktionen in verschiedenen Kontexten bewegen (beispielsweise als staatliche Delegierte bei internationalen Verhandlungen, als aktives Mitglied einer Nichtregierungsorganisation und als Vorsitzende eines Gremiums der Vereinten Nationen) könnten hier Handlungsspielräume schaffen und als GrenzgängerInnen auftreten. Institutionen sind in dieser Hinsicht räumliche Felder der Kontestation, die NormunternehmerInnen dazu nutzen, alternative Vorschläge zur Gestaltung gemeinschaftlicher Beziehungen (z.B. die angemessene Verteilung von prozeduralen und substanziellen Rechten und Pflichten für bestimmte Akteursgruppen innerhalb einer Institution) jenseits des status quo zu entwickeln, vorzubringen und zu verhandeln. Hier schließt sich also die Frage an, auf der Grundlage welcher Wissensbe-

15 Der Begriff des Feldes wurde bereits von Bourdieu zur Beschreibung relationaler Beziehungen verwendet (Bourdieu 1998: 48-52). Allerdings entsteht der physische Raum bei Bourdieu nicht durch Anordnung, sondern er ist vielmehr Ausdruck der relationalen Anordnungen des sozialen Raums (Löw 2001: 182). Vgl. auch das »organisatorische Feld« bei DiMaggio/Powell (1983).

16 Vgl. der "gelebte Raum« bei Lefebvre (2006 [1974]: 336); und die "gesellschaftlichen Raumverhältnisse" bei Belina/Michel (2008: 25).

17 Während die Rede vom Management institutioneller Interaktion impliziert, dass ein zielgerichtetes und bewusstes Handeln vorliegt, ist es aus raumsoziologischer Sicht auch möglich, dass unbewusstes Handeln oder Handeln unter Bedingungen von Nicht-Wissen oder selektiver Wahrnehmung Raum-konstituierend wirkt. Gemein ist beiden Perspektiven allerdings, dass nicht-intendierte Handlungsfolgen grundsätzlich berücksichtigt werden können. 
stände Verknüpfungen zwischen den Institutionen absichtsvoll hergestellt werden. ${ }^{18}$ So geht es bei einer Analyse normativen Wandels im internationalen System, die auf die Bedeutung institutioneller Interaktion fokussiert, um die Untersuchung von Spannungen und Grenzveränderungen, die durch materielle und immaterielle, formale und informelle Wechselwirkungen zwischen Räumen entstehen. Durch das Management solcher Interaktionen tragen AkteurInnen - so die Argumentationslinie - zur Gestaltung neuer Räume bei. Wie Oran Young formuliert: »Institutional interplay becomes a matter of politics when actors engaged in specific interactions seek consciously to make use of overlaps to achieve identifiable goals« (Young 2002: 137). Erst durch diese analytische Wendung ist es möglich, unterschiedliche - und doch gleichzeitig wirkende - Ausprägungen von Macht (Barnett/Duvall 2005; Okereke et al. 2009) zu erfassen, die die Wechselwirkung zwischen Institutionen gestalten und gleichzeitig von dieser geprägt werden. Das (Neu-)Design von Institutionen ist dann nicht mehr primär als Ergebnis Effizienz-orientierter Bemühungen oder der konsistenten Übertragung/Anwendung von Normen und Prozeduren zu betrachten, sondern als Resultat von politics (inklusive nicht-intendierter Handlungsfolgen): Raum und damit auch Wechselbeziehungen institutioneller Interaktion sind per se politisch.

Zusammenfassend wird argumentiert, dass die Interdependenz internationaler Institutionen durch soziale Wechselbeziehungen konstituiert ist und ständig rekonfiguriert wird. Die Perspektive auf institutionelle Interaktion und Normwandel verharrt somit nicht bei einer deskriptiven Bestandsaufnahme zur Frage, in welchen räumlichen Positionen und Mandatsverhältnissen sich AkteurInnen befinden. Sie fragt hingegen, wie Akteure jene relationalen Räume aktiv konstituieren, um hierdurch alternative (diskursive) und emanzipatorische Machtressourcen zu generieren, d.h. ihre relativen Interessen besser zu artikulieren, durchzusetzen und Normwandel voran zu treiben. Hierdurch wird institutionelle Interaktion, beispielsweise in multilateralen Verhandlungen überhaupt erst hervorgebracht. Die Produktion und Gestaltung inter-institutioneller Strukturen sind dabei umkämpft. So interessiert in der hier gewählten Perspektive also vor allem die (plausible) Ursächlichkeit diskursiver Räumlichkeit für

18 Hier wird noch einmal deutlich, dass die AkteurInnen, die durch eine solche raumsoziologische Perspektive in den Blick genommen werden können, immer schon über eine SprecherInnenposition verfügen (und damit nicht der Gruppe der Subalternen zugehören). In anderen Worten: Der Zugang zu den sozialen Gütern, die angeordnet bzw. in Beziehung zueinander gesetzt werden sollen, ist Voraussetzung für die Möglichkeit, an der Gestaltung von Räumen beteiligt zu sein. 
politischen Wandel. Denn nur »wenn der Raumbegriff selbst und nicht nur das Handeln als bewegt gefasst wird, [...] [können] auch Veränderungen von Räumen verstanden werden« (Löw 2001: 65).

\subsection{Raum, Relation und Sprache}

So wirken Raum und Zeit nicht in einem rationalistischen Sinn ausschließlich a priori. Zwar müssen sich AkteurInnen immer auch zu einer objektivierten Raum-Zeit-Struktur verhalten (Harvey 2008 [1990]). ${ }^{19}$ Allerdings können die scheinbar aprioristischen historisch, kulturell, sozial bedingten Grundstrukturen stets re- und dekonstruktiert werden, wodurch ihr relativistischer und idealer Gehalt hervordringt. Raum und Zeit werden zu sozialen Konstrukten. Gleichzeitig scheinen sie dem oder der jeweils betroffenen (sprechenden) AkteurIn möglicherweise unhintergehbar, weil sie mit seiner Identität untrennbar verwoben sind und sozial differenzierend, also relativierend wirken (zum letzten Punkt Harvey 2008 [1990]: 37). Die Selbst- und Fremdbeschreibung bzw. die Attribuierung einer bestimmten Akteursgruppe generiert »a new social kind « (Barnett/ Duvall 2005: 61). Die jedem Sprechakt implizite Selbstbeschreibung ist demnach (über Sprache, Text, symbolische Artefakte) auch identitätsstiftende Selbstsymbolisierung (Drepper 2003: 112). In jedem Fall müssen die Elemente einer retrospektiven Projektion, aus der sich eine Identitätsdarstellung immer nur speisen kann, tatsächlich artikuliert, also öffentlich benannt und dargestellt werden, um die ihnen zugrunde liegende Relativität der Bezugssysteme ausdrücken zu können. ${ }^{20}$ Insofern können sich durch Bezugnahme auf historische Ereignisse und Narrative auch diskursive Pfadabhängigkeiten konkretisieren.

Zudem ist die Anschlussfähigkeit von Deutungsmustern nicht absolut, sondern stets vermittelt, beispielsweise durch die Erwartungshaltung, die aus der faktischen Hierarchie sozialer Strukturen erwächst (vgl. Drepper 2003: 111). Letzten Endes existieren Selbstbeobachtungen und Selbstbeschreibungen "nur im Ereigniszusammenhang des Systems" (Luhmann

19 Die scheinbar allgemeingültige (verobjektivierte) Strukturierung des gesellschaftlichen Raum-Zeit-Struktur kann selbst aber auch wiederum Gegenstand von Kritik und Wandel sein, so bei Rosa (2005).

20 Vgl. die von Lacan inspirierte Annahme Bhabhas, dass eine Individualisierung des Subjekts als Handelnder erst retroaktiv bzw. durch Nachträglichkeit entsteht, wenn es seinen Platz in der symbolischen Ordnung eingenommen hat und aufgefordert wurde zu sprechen (in: Do Mar Castro Varela/Dhawan 2005: 98). 
1997: 883). ${ }^{21}$ Die Selbstbeobachtung und ihr Ausdruck in Sprache - durch die wiederum die faktische Existenz hierarchischer Sozialbeziehungen und damit Identität konstituiert wird - funktioniert also nicht ohne den normativen und performativen systemischen Bezugspunkt (der auch als Gesamtheit aller anderen Elemente gesehen werden kann). So erfolgt die Formulierung von bestimmten sozialen und politischen Forderungen stets aus der SprecherInnenposition innerhalb einer hierarchisierten Ordnungsstruktur. Beide ergeben sich letzten Endes nur aus dem Anderen, bilden gemeinsam schließlich den sozialen Raum.

In einer solchen diskurstheoretischen Lesart ist die Bildung sozialer Räume unauflöslich mit der sprachlichen Anordnung von Subjektpositionen (spacing) und wechselseitiger Bedeutungszuschreibung (Syntheseleistung) verbunden. Institutionen und die in ihnen geronnenen Diskurse heben nach diesem Verständnis somit die intellektualistische Unterscheidung von Sprechen und Handeln auf (vgl. hierzu Krämer 2001). In dieser Lesart entsteht ein diskursiver Raum durch die Gleichzeitigkeit von Platzierung (von Körpern) und Verknüpfung (Relationenbildung). Hieraus folgt das Argument, dass die Wechselbeziehungen internationaler Institutionen nur durch versprachlichte relationale Räume angemessen erfasst werden können. Sie sind letzten Endes durch gezieltes sprachliches Handeln konstituiert. So ist es nicht angemessen, einen relationalen Raum auf die Topologie seiner materiellen Elemente zu reduzieren. Vielmehr geht es um ein räumliches Bewegungsfeld, in dem sich die Träger materieller und nicht-materieller Eigenschaften und Darstellungen (auch Symbole) zueinander und über Organisationsgrenzen hinweg positionieren, sich selbst und andere als Subjekte definieren. In der Konsequenz geht es somit zum einen darum, den oder die sprachbegabte(n) AkteurIn im Raum bzw. in der Raumzeit als wesentlichen »Träger der Realität« (Günzel 2006: 40) anzusehen. Zum anderen sollen aber auch die durch die sprachlichen Handlungen des oder der AkteurIn hervorgerufenen Bewegungen der Räume selbst (diskursive Konstitution/Verknüpfung, Verlagerung/Ausschluss etc.) erfasst werden. ${ }^{22}$ So werden die normativen wie auch pragmatischen Grundlagen formaler Ordnungsstruktur durch Politisierung bzw. durch

21 Ähnlich die »verkörperte Sprache« der »asymmetrisch positionierten Körperwesen« bei Krämer (2001: 270).

22 Nicht-substanzialistisch bzw. nicht-empiristisch ist mein Zugriff dabei lediglich in dem Sinne, dass er nicht nur auf die physikalische Welt (und ihre örtlichen Räume) fokussiert. In einem kartesischen Sinne, wonach das Denkende (res cogitans) ideelle Substanz ist, wäre er sehr wohl als solcher zu bezeichnen - ein Raum kann demnach per definitionem nicht 'leer' sein. Erst vor diesem Hintergrund ist 
inter-institutionelle kommunikative Möglichkeitsräume herausgefordert. ${ }^{23}$ AkteurInnen können dabei aus dem sprachlichen und materiellen Reservoir des spezifischen formalen Raums schöpfen, können diesen aber auch transzendieren, indem sie beispielsweise auf Erfahrungen und Argumente aus anderen organisationalen Kontexten zurückgreifen. So konstituiert sich der Raum der Interaktionsbeziehungen zwischen unterschiedlichen Institutionen/Organisationen in der Gesamtschau erst durch aktive Verknüpfungen der AgentInnen, die sich unter vorstrukturierten Bedingungen zusammenfinden (vgl. Löw 2001: 158). Die Zusammenschau dieser Dimensionen konkretisiert die räumliche Struktur einer Organisation letzten Endes als Wirklichkeit (Lefebvre 2006 [1974]: 336). Insofern wäre die rationalistische Rede von der Überlappung internationaler Institutionen die statisch-topologische Beschreibung für einen relationalen Raum, der gleichwohl erst aus den sozialen und argumentativen Operationen zur Platzierung und Verbindung von Gütern und AkteurInnen (Subjekt- und SprecherInnenpositionen) entsteht.

Diese Prozesse entwickeln sich nicht zuletzt in internationalen Verhandlungen. Das durch Verhandlungen bestimmte (Neu-)Design internationaler Institutionen - und der damit evtl. einhergehende Normwandel kann somit als Raum(re-)produktion konzipiert werden.

\subsection{Die Räumlichkeit internationaler Verhandlungen in den Vereinten Nationen}

Ein Beispiel hierfür sind die Vereinten Nationen (VN), die einen relationalen Raum darstellen, in dem materielle und symbolische Güter und AkteurInnen in einer spezifischen Art angeordnet werden und diese Strukturen selbst im Handeln (re-)produzieren. Territorial-räumliche Bezüge der VN bestehen nicht nur hinsichtlich der Zielkontexte ihrer Programmumsetzung, sondern auch bezüglich der Orte und Gebäude ihrer Hauptquartiere und der Veranstaltungsorte von VN-Verhandlungen. Gleichzeitig ent-

es möglich, mich einerseits vom empiristischen spatial turn abzugrenzen und meinen Ansatz andererseits der phänomenologischen Erfassung von Räumlichkeit zuzuordnen. Physische Gesetzmäßigkeiten und physikalische Ereignisse wirken in dieser Lesart vor allem als »Bildhintergrund (Lefebvre 2006 [1974]: 330). Sie haben über soziale und kulturelle Vermittlung unterschiedliche Effekte für verschiedene gesellschaftliche Gruppen. Über Verletzlichkeit und Anpassungsfähigkeit sind natürliche und soziale Räume verbunden (vgl. Wallbott 2012).

23 Zur räumlichen Dimension von Widerstand auch Castells (1983: 4). 
wickelt sich eine deterritoriale Räumlichkeit in dem Sinne, dass die Anordnungen und Verknüpfungen in grenzüberschreitenden Regeln eingeschrieben und durch Ressourcen abgesichert sind (vgl. die Ausführungen zum Strukturbegriff in Löw 2001: 171). In den Gremien der VN, in ihren Unterprogrammen und Konventionen verbinden sich je eine normative Grundausrichtung und spezifische inhaltliche Programmatik mit einem bestimmten bürokratischen Apparat. Staatliche wie nicht-staatliche AkteurInnen, die innerhalb dieses Raums mit bestimmten Kompetenzen wie z.B. formalen Zugangsrechten ausgestattet (angeordnet) sind, begegnen sich hier. Gleichzeitig konstituieren die unterschiedlichen AkteurInnen den Raum der VN durch sprachliche Äußerungen und Handlungen, indem sie sich attribuieren und den ihnen (selbst- und fremd-) zugeschrieben Habitus (diplomatisches Verhandeln, Kritik, Beratung, Agenda-Formulierung, Sitzungsleitung und Konsultation etc.) realisieren. So nehmen sich die Mitgliedstaaten, Nicht-Regierungsorganisationen, die Verhandlungsleiter, das Sekretariat usw. wechselseitig wahr als derart situierte/ angeordnete AkteurInnen (Syntheseleistung).

Nach innen wie nach außen wird der Raum der VN darüber hinaus durch ein Konglomerat konzeptioneller Symbole und Codes repräsentiert. Nach innen wirken beispielsweise intertextuelle Bezüge - z.B. Verweise auf frühere Resolutionen - sowie diplomatische Rhetorik und Habitus ritualisierend und kohärenzsteigernd. Nach außen repräsentieren die Flagge und Eröffnungs- und Schlusszeremonien bei großen Konferenzen den Raum der VN, ebenso wie der wiederkehrende sprachliche Aufbau offizieller Dokumente und ihre spezifische Codierung. Zudem agieren und bewegen sich AkteurInnen mit den ihnen eigenen Symbolen in diesem Raum. Durch Bedeutungszuschreibungen von Teilnehmern und Beobachtern sowie repetitive Handlungs-Routinen wird somit ein bestimmtes Bild der VN konstruiert und reproduziert (vgl. beispielsweise die Verhandlung der Rechte indigener Gruppen unter Abgrenzung zu lokalen Gemeinschaften im Zusammenhang mit der Wald- und Klimapolitik der VN sowie den Wandel der Bedeutung von Indigenität; hierzu Wallbott 2016; Wallbott 2019).

Dass der organisationale Raum der VN, gekennzeichnet durch die formale Gleichstellung seiner staatlichen Mitglieder, erst durch bestimmte relationale Wahrnehmungsstrukturen z.B. der Über- und Unterordnung der Mitglieder sowie Sprechakte komplettiert wird (vgl. auch Barnett/Duvall 2005: 52; Towns 2012), kann sehr knapp am Beispiel der Afrikanischen Gruppe in den Verhandlungen zum Nagoya-Protokoll illustriert werden. Das Protokoll ist ein Instrument der Konvention zur Biologischen Vielfalt (CBD) der VN, wurde 2010 verabschiedet und regelt den Zugang zu gene- 
tischen Ressourcen und die Verteilung der Gewinne, die aus ihrer Nutzung erfolgen (vgl. Wallbott et al. 2014). In den Verhandlungen forderte die Afrikanische Gruppe, an solchen Gewinnen beteiligt zu werden, die durch Nutzung derjenigen natürlichen Ressourcen entstehen, die in Kolonialzeiten entnommen worden waren (und fortan ex situ gelagert wurden, beispielsweise in Botanischen Gärten oder Gendatenbanken). ${ }^{24}$ Die Selbstbeschreibung aus einer als schwach gerahmten Verhandlungsposition heraus (wenig Ressourcen, Betroffene kolonialer Ausbeutung) vermittelt das Bild eines faktisch hierarchischen internationalen Staatensystems, getragen durch das Bewusstsein für dessen historische Bedingtheit, die relative Wahrnehmung des zeitlichen Nachhalls zurückliegender Ereignisse und deren Wirkmächtigkeit im kollektiven Gedächtnis der Unterworfenen. Die Ent-Kolonialisierung hat zwar einerseits die strukturell-systemische Position der ehemaligen Kolonien verändert. Andererseits wirken die wahrgenommenen Über- und Unterordnungsverhältnisse der Kolonialzeit und die während dieser Zeit geschaffenen Fakten (z.B. entnommene Ressourcen) aber im interpretativen System - »through which they understand their interests and desires " (Barnett/Duvall 2005: 54) - und in den Argumentationsmustern der jungen Nationalstaaten in einer Art kontinuierlicher "Geschichtlichkeit“" (Weidenhaus 2015) nach. ${ }^{25}$

Es kann argumentiert werden, dass die Anerkennung dieser Forderung eine Ausweitung des normativen Kernbestands der Konvention um eine Norm historischer Verantwortung bedeutet hätte. Dabei wurde die Argumentation der Afrikanischen Gruppe wesentlich unterstützt durch gezieltes Interdependenz-Management u.a. zwischen der CBD und der Weltorganisation für geistiges Eigentum oder der Weltgesundheitsorganisation aber auch zwischen der CBD und panafrikanischen institutionellen Räumen, in denen sich die Mitglieder der Gruppe über den Reichweite und Bedeutung der relevanten Normen verständigten (für eine detailliertere

24 Hier zeigt sich bereits, dass auch ein relationaler Raumbegriff nicht als Ersatz, sondern vielmehr als Ergänzung zu einem geographischen Raumbegriff zu sehen ist. So verbindet die Afrikanische Gruppe in ihrer Argumentation die Problematisierung territorialer und funktionaler Souveränität bzw. deren historische Kontingenz mit einer real-örtlichen in situ/ex situ-Dichotomie zur Kategorisierung von Verhandlungsgegenständen.

25 Eine angemessene Beantwortung der Frage, ob die Identifizierung der eigenen strukturalen Position im Falle der Afrikanischen Gruppe in den Nagoya-Verhandlungen zu Resignation oder Akzeptanz des hierarchischen Verhältnisses führte, wie es eine neo-marxistische Rede von der Wirksamkeit struktureller Macht implizieren würde, kann an dieser Stelle aus Platzgründen nicht geleistet werden. 
Betrachtung des Verhandlungsverlaufs und -ergebnisses vgl. Wallbott 2014a).

\section{Schluss}

In diesem forschungspragmatischen Beitrag wurde ein prozessualer und relationaler Raumbegriff als konzeptionelle und analytische Ergänzung zur Normenforschung und zum Forschungsprogramm zur institutionellen Interaktion eingeführt. Insbesondere letzteres befasste sich bisher recht formalistisch und funktionalistisch mit den Effekten einer fragmentierten globalen Polit-Architektur. Aber Wechselbeziehungen zwischen internationalen Institutionen finden eben nicht nur technokratisch-rational in präformierten Räumen statt. Und ebenso wenig erschöpft sich das analytische Potential des Forschungsprogramms zur institutionellen Interaktion in der Untersuchung manifester Konflikte zwischen verschiedenen Ordnungsstrukturen.

Vielmehr lohnt darüberhinausgehend die Untersuchung der Frage, wie AkteurInnen die Wechselwirkungen zwischen Institutionen politisieren, indem sie einen inter-institutionellen Argumentationsraum konstruieren, in dem sie Forderungen an die prozedurale und substanzielle Gestaltung institutioneller Ordnungen formulieren. Daher geht der in diesem Beitrag gewählte Raumbegriff über strukturalistische Systemansätze hinaus und integriert soziale, materielle, diskursive und ideelle Dimensionen von Räumlichkeit. So wird die intentionale Verbindung von Diskursivität, Symbolik und Materialität unterschiedlicher Räume dezidiert als Vehikel für die Verlautbarung und das Sichtbarmachen von Kritik und die Forderung nach Veränderung betrachtet. Denn Raum ist »nicht gegeben, sondern wird durch die Interaktion von Raumkörpern oder menschlichen Handlungen bestimmt (Günzel 2006: 41; Belina/Michel 2008: 8). Die Bedingungen der Entstehung und Neukonfiguration räumlicher Strukturen durch aktive Ordnungs- und Verknüpfungsleistungen unterschiedlicher Akteursgruppen wird hierdurch zum Forschungsgegenstand. So ist zu untersuchen, wie AkteurInnen zur Konstituierung, zum Aufbau und zur Gestaltung von Räumen beitragen und wie sich hierdurch bestehende organisationale Grenzen verschieben. Der sich aus solch strategischem Handeln möglicherweise ergebende Normwandel wird also nicht als output einer abgeschlossenen Teilstruktur des fragmentierten Gesamtsystems betrachtet, sondern als Ergebnis ebenso wie Ausgangsbedingung der wechselseitigen Beeinflussung von Teilsystemen. 
Dabei ist im Unterschied zur bisherigen Ausrichtung der neo-institutionalistischen Forschung nicht davon auszugehen, dass das strategische Management von Räumen und räumlichen Strukturen ausschließlich den bereits Mächtigen als Medium vorbehalten ist. Auch nicht-hegemoniale AkteurInnen sind potenziell in der Lage, Räume zu konstruieren und hierdurch ihre produktive diskursive Macht zu realisieren. Ebenso wenig allerdings sollte der Historizität von Strukturen und Machtbalancen jede Bedeutung abgesprochen werden. Im Gegenteil: Die Frage »ob Geschichte/ Zeit oder Geographie/Raum prioritär seien « (Belina/Michel 2008: 23) ist meines Erachtens wenig zielführend, um soziale Prozesse und politische Entwicklungen zu erklären. Vielmehr geht es um eine Verbindung dieser beiden Dimensionen in der Hinsicht, dass institutionelle Räume eben immer gleichzeitig sozial und diskursiv konstruiert wie historisch kontingent und stets unabgeschlossen sind. Sie sind als ständig rekonfigurierte Struktur die Vorbedingung von Politik und somit untrennbar »in die Produktion von Geschichte verwickelt" (Massey 2008 [1992]: 117). Um diesen Ansatz, der wesentliche Elemente kritischer Normen- und Institutionalismusforschung vereint, weithin fruchtbar zu machen, möchte ich abschließend mögliche zukünftige kausalanalytische und normative Forschungsperspektiven skizzieren, wobei die Reflektion der eigenen Vorannahmen und Situiertheit der Forschenden stets mitbedacht werden sollten (vgl. die grundlegenden Ausführungen von Loges in diesem Band).

Zunächst ist auf Grundlage dieses Beitrags für die weitere empirische Forschung interessant, inwieweit Strategien der gezielten Delegitimierung und Kooptation bestimmter Akteursgruppen über verschiedene Räume hinweg gezielt koordiniert werden und wie sich verschiedene Institutionen bzw. die in ihnen handelnden AkteurInnen hinsichtlich der Öffnung oder Schließung für unterschiedliche Anspruchsgruppen beeinflussen. Diese Prozesse der Verengung von Diskursräumen könnten dann wiederum mit Analysen zur Ausweitung von Handlungsspielräumen in Beziehung gesetzt werden, um die Dynamik institutioneller Wechselwirkungen und Grenzziehungen zu voice und vote in Gänze zu erfassen.

Auch lässt der hier entwickelte Ansatz Raum für die kritisch-konstruktivistische Konnotation, dass Normen eine dynamische Qualität in der Hinsicht besitzen, dass Bedeutungszuschreibungen kontextabhängig und somit variabel sind (Wiener/Puetter 2009; Wiener 2008). So lässt sich die Untersuchungsfrage in die aktuelle Normenforschung einbinden, inwieweit die Bezugnahme auf argumentative Ressourcen aus anderen Teilordnungen der Global Governance-Architektur mit einem Bedeutungswandel dieser Norm einhergeht. Eine gehaltvolle normativ-theoretische Wendung kann die Forschung zur institutionellen Interaktion also nehmen, wenn 
sie fragt, wie Bedeutung im Prozess der institutionellen Wechselwirkung durch Syntheseleistung generiert wird, wie Normen in inter-institutionellen Räumen von welchen AkteurInnen mit welcher Autorität und auf der Grundlage welcher Interessen mit Bedeutung (neu) belegt werden und welche Spannungsverhältnisse sich hierbei zwischen der traditionellen Ausrichtung der sogenannten Zielorganisation und der Bedeutung der Norm in der Ursprungsorganisation unter Umständen ergeben (Wallbott 2016). Wie gestalten sich beispielsweise Aushandlungsprozesse, wenn die Norm zu Rechten indigener Völker in die Klimaverhandlungen der Vereinten Nationen eingebracht wird und auf die technokratischen Paradigmen internationaler Umweltpolitik trifft (Wallbott 2016)? Welche raumbildenden Dynamiken ergeben sich durch das Aufeinandertreffen symbolisch und narrativ unterschiedlicher Traditionen? Wie wirken sich Auslassungen und Selektivitäten auf die Robustheit von Normen aus? Darüber hinaus ist zu erörtern, was Normwandel, der durch institutionelles Interdependenz-Management angestoßen wurde, für die Legitimität und Effektivität von Regime-Komplexen bedeutet.

Schließlich kann die kritische Normenforschung wiederum von der vorgeschlagenen Agenda profitieren, da hierdurch die Auswirkungen unterschiedlicher professioneller Prägungen in den jeweiligen Teilordnungen für die Bedeutungszuschreibung in den Blick genommen werden (hierzu auch Wiener 2007: 12). In dieser Richtung verweist die Frage nach der Relevanz von biographischen Wissensbeständen und beruflichen Fachkulturen in Prozessen institutioneller Wechselwirkung auf einen möglichen analytischen Mehrwert (Wallbott 2014c), ebenso wie die Beantwortung der Frage, nach welchen Relevanzkriterien unterschiedliche kollektive Wissensbestände (z.B. traditionelles Wissen) über organisationale Grenzen hinweg erfolgreich mobilisiert werden.

\section{Literatur}

Barnett, Michael/Duvall, Raymond 2005: Power in International Relations, in: International Organization 59: 1, 39-75.

Belina, Bernd/Michel, Boris 2008: Raumproduktionen, in: Belina, Bernd/Michel, Boris (Hrsg.): Raumproduktionen. Beiträge der Radical Geography. Eine Zwischenbilanz, Münster, 7-34.

Bell, Stephen 2011: Do We Really Need a New 'Constructivist Institutionalism' to Explain Institutional Change? In: British Journal of Political Science 41: 4, 883906. 
Benvenisti, Eyal/Downs, George W. 2007: The Empire's New Clothes: Political Economy and the Fragmentation of International Law, in: Stanford Law Review 60: 2, 595-631.

Biermann, Frank/Betsill, Michele M./Gupta, Joyeeta/Kanie, Norichika/Lebel, Louis/ Liverman, Diana/Schroeder, Heike/Siebenhüner, Bernd 2009: Earth System Governance: People, Places and the Planet: Science and Implementation Plan of the Earth System Governance Project, Bonn.

Biermann, Frank/Zelli, Fariborz/Pattberg, Philipp/van Asselt, Harro 2010: The Architecture of Global Climate Governance: Setting the Stage, in: Biermann, Frank/ Pattberg, Philipp/Zelli, Fariborz (Hrsg.): Global Climate Governance Beyond 2012, Cambridge, MA, 15-24.

Biermann, Frank/Siebenhüner, Bernd/Bauer, Steffen/Busch, Per-Olof/Campe, Sabine/ Dingwerth, Klaus/Grothmann, Torsten/Marschinski, Robert/Tarradell, Mireia 2009: Studying the Influence of International Bureaucracies: A Conceptual Framework, in: Biermann, Frank/Siebenhüner, Bernd (Hrsg.): Managers of Global Change: The Influence of International Environmental Bureaucracies, Cambridge, MA, 15-74.

Bourdieu, Pierre 1998: Praktische Vernunft. Zur Theorie des Handelns, Frankfurt a. M..

Braithwaite, John/Drahos, Peter 2000: Global Business Regulation, Cambridge, MA.

Breitmeier, Helmut/Underdal, Arild/Young, Oran 2011: The Effectiveness of International Environmental Regimes: Comparing and Contrasting Findings from Quantitative Research, in: International Studies Review, 13: 4, 579-605.

Bürk, Thomas. 2006: Raumtheoretische Positionen in angloamerikanischen und deutschsprachigen sozial- und kulturwissenschaftlichen Publikationen seit 1997. Ein Literaturbericht. http://www.forschungsnetzwerk.at/downloadpub/lit-raumt heorie.pdf.

Busch, Marc L. 2007: Overlapping Institutions, Forum Shopping, and Dispute Settlement in International Trade, in: International Organization 61, 735-61.

Busch, Per-Olof/Jörgens, Helge 2007: Dezentrale Politikkoordination im internationalen System - Ursachen, Mechanismen und Wirkungen der internationalen Diffusion politischer Innovationen, in: Holzinger, Katharina/ Jörgens, Helge/ Knill, Christoph (Hrsg.): Transfer, Diffusion und Konvergenz von Politiken (Politische Vierteljahresschrift Sonderheft 38), 56-84.

Castells, Manuel 1983: The City and the Grassroots, London.

Coleman, Katharina P. 2013: Locating Norm Diplomacy: Venue Change in International Norm Negotiations, in: European Journal of International Relations 19: 6, 163-186.

Daase, Christopher/Deitelhoff, Nicole 2014: Zur Rekonstruktion globaler Herrschaft aus dem Widerstand. Internationale Dissidenz Working Paper 1, Frankfurt a.M..

De Certeau, Michel 2006 [1980]: Praktiken im Raum, in: Dünne, Jörg/Günzel, Stephan (Hrsg.): Raumtheorie. Grundlagentexte aus Philosophie und Kulturwissenschaften, Frankfurt a. M., 343-353. 
Deitelhoff, Nicole 2006: Überzeugung in der Politik. Grundzüge einer Diskurstheorie internationalen Regierens, Frankfurt a. M..

DiMaggio, Paul J. /Powell, Walter W. 1983: The Iron Cage Revisited: Institutional Isomorphism and Collective Rationality in Organizational Fields, in: American Sociological Review 48: 2, 147-160.

Do Mar Castro Varela, María/Dhawan, Nikita 2005: Postkoloniale Theorie. Eine kritische Einführung, Bielefeld.

Drepper, Thomas 2003: Organisationen der Gesellschaft, Wiesbaden.

Drezner, Daniel W. 2009: The Power and Peril of International Regime Complexity, in: Perspectives on Politics 7: 1, 65-70.

Finnemore, Martha/Sikkink, Kathryn 1998: International Norm Dynamics and Political Change, in: International Organization 52: 4, 887-917.

Freistein, Katja/Liste, Philip 2011: "Ever-always-the-same": An Intertextual Approach towards International Institutions and the Politics of Foundation, unv. Ms..

Gehring, Thomas 2007: Einflussbeziehungen zwischen internationalen Institutionen im Spannungsfeld von Handel und Umwelt. Von gegenseitiger Störung zur institutionalisierten Arbeitsteilung zwischen internationalen Umweltinstitutionen und der Welthandelsorganisation, in: Jacob, Klaus/Biermann, Frank/Busch, PerOlof/Feindt, Peter H. (Hrsg.): Politik und Umwelt (Politische Vierteljahresschrift Sonderheft 39), Wiesbaden, 94-114.

Gebring, Thomas/Oberthür, Sebastian 2009: The Causal Mechanisms of Interaction between International Institutions, in: European Journal of International Relations 15: 1, 125-156.

Gebring, Thomas/Oberthür, Sebastian 2011: Institutional Interaction: Ten Years of Scholarly Development, in: Oberthür/Stokke 2011, 25-58.

Gruber, Lloyd 2000: Ruling the World: Power Politics and the Rise of Supranational Institutions, Princeton, NJ.

Günzel, Stephan 2006: Physik und Metaphysik des Raums. Einleitung, in: Dünne, Jörg/Günzel, Stephan (Hrsg.): Raumtheorie. Grundlagentexte aus Philosophie und Kulturwissenschaften. Frankfurt a. M., 19-93.

Günzel, Stephan 2008: Spatial Turn - Topographical Turn - Topological Turn. Über die Unterschiede zwischen Raumparadigmen, in: Döring, Jörg /Thielmann, Tristan (Hrsg.): Spatial Turn. Das Raumparadigma in den Kultur- und Sozialwissenschaften, Bielefeld, 219-237.

Haas, Peter M./Levy, Marc A./Keohane, Robert. O. (Hrsg.) 1993: Institutions for the Earth: Sources of Effective International Environmental Protection, Cambridge, MA.

Harvey, David 1973: Social Justice and the City, Oxford.

Harvey, David 2008 [1990]: Zwischen Raum und Zeit: Reflektionen zur Geographischen Imagination, in: Belina, Bernd/Michel, Boris (Hrsg.): Raumproduktionen. Beiträge der Radical Geography. Eine Zwischenbilanz, Münster, 36-60.

Helfer, Laurence R. 2004: Regime Shifting: The TRIPS Agreement and New Dynamics of International Intellectual Property Lawmaking, in: The Yale Journal of International Law 29: 1, 1-83. 
Holzscheiter, Anna, 2005: Discourse as Capability: Non-State Actors' Capital in Global Governance, in: Millennium - Journal of International Studies 33, 723-746.

Holzinger, Katharina/Jörgens, Helge/Knill, Christoph 2007. Transfer, Diffusion und Konvergenz. Konzepte und Kausalmechanismen, in: Holzinger, Katharina/ Jörgens, Helge/Knill, Christoph (Hrsg.): Transfer, Diffusion und Konvergenz von Politiken (Politische Vierteljahresschrift Sonderheft 38), Wiesbaden, 11-35.

Keim, Karl-Dieter 2003: Das Fenster zum Raum. Traktat über die Erforschung sozialräumlicher Transformation, Opladen.

Keohane, Robert O. 1989: Neoliberal Institutionalism: A Perspective on World Politics, in: Keohane, Robert O. (Hrsg.): International Institutions and State Power: Essays in International Relations Theory, Boulder, CO, 1-20.

Koremenos, Barbara/Lipson, Charles/Snidal, Duncan (Hrsg.) 2004: The Rational Design of International Institutions, Cambridge, MA.

Krämer, Sybille 2001: Sprache, Sprechakt, Kommunikation. Sprachtheoretische Positionen des 20. Jahrhunderts, Frankfurt a. M..

Krasner, Stephen 1983: Structural Causes and Regime Consequences: Regimes as Intervening Variables, in: Krasner, Stephen (Hrsg.): International Regimes, Ithaca, NY, 1-21.

Laclau, Ernesto 1990: New Reflections on the Revolution of Our Time, London.

Lefebvre, Henri 2006 [1974]: Die Produktion des Raums, in: Dünne, Jörg/Günzel, Stephan (Hrsg.): Raumtheorie. Grundlagentexte aus Philosophie und Kulturwissenschaften Frankfurt a. M., 330-342.

Lubmann, Niklas 1997: Die Gesellschaft der Gesellschaft, Frankfurt a. M..

Löw, Martina 2001: Raumsoziologie, Frankfurt a. M..

Massey, Doreen 2008 [1992]: Politik und Raum/Zeit, in: Belina, Bernd/Michel, Boris (Hrsg.): Raumproduktionen. Beiträge der Radical Geography. Eine Zwischenbilanz, Münster, 111-132.

Mayntz, Renate/Scharpf, Fritz W. 1995: Der Ansatz des akteurszentrierten Institutionalismus, in: Mayntz, Renate/Scharpf, Fritz W. (Hrsg.): Gesellschaftliche Selbstregelung und politische Steuerung. Frankfurt a. M., 39-72.

Merleau-Ponty, Maurice 2003 [1961]: Das Auge und der Geist. Philosophische Essays, Hamburg.

Miles, Edward L./Underdal, Arild/Andresen, Steinar/Wettestad, Jørgen/Skjorseth, Jon Birger/ Carlin, Elaine M. 2002: Environmental Regime Effectiveness: Confronting Theory with Evidence, Cambridge, MA.

Oberthür, Sebastian 2009: Interplay Management: Enhancing Environmental Policy Integration among International Institutions, in: International Environmental Agreements: Politics, Law and Economics 9: 4, 371-391.

Oberthür, Sebastian/Stokke, Olav Schram (Hrsg.) 2011a: Managing Institutional Complexity: Regime Interplay and Global Environmental Change, Cambridge, MA.

Oberthür, Sebastian/Stokke, Olav Schram 2011b: Introduction: Institutional Interaction in Global Environmental Change, in: Oberthür/Stokke 2011a, 1-23. 
Oberthür, Sebastian/Stokke, Olav Schram 2011c: Conclusions: Decentralized Interplay Management in an Evolving Interinstitutional Order, in: Oberthür/Stokke 2011a, 313-341.

Okereke, Chukwumerije /Bulkeley Harriet/Schroeder Heike 2009: Conceptualizing Climate Change Governance Beyond the International Regime, in: Global Environmental Politics 9: 1, 58-78.

Ostrom, Elinor 2010: Polycentric Systems for Coping with Collective Action and Global Environmental Change, in: Global Environmental Change 20: 4, 550-557.

Pollack, Mark 2003: Engines of European Integration: Delegation, Agency, and Agenda Setting in the EU, New York, NY.

Rosa, Hartmut 2005: Beschleunigung. Die Veränderung der Zeitstrukturen in der Moderne, Frankfurt a. M..

Rosendal, G. Kristin 2001a: Impacts of Overlapping International Regimes: The Case of Biodiversity, in: Global Governance 7, 95-117.

Rosendal, G. Kristin 2001b: Overlapping International Regimes: The Case of the Intergovernmental Forum on Forests (IFF) between Climate Change and Biodiversity, in: International Environmental Agreements 1: 4, 447-468.

Scharpf, Fritz W. 2000: Interaktionsformen. Akteurzentrierter Institutionalismus in der Politikforschung, Opladen.

Schmidt, Vivien 2008: Discursive Institutionalism: The Explanatory Power of Ideas and Discourse, in Annual Review of Political Science 11, 303-326.

Schmidt, Vivien 2010: Taking Ideas and Discourses Seriously: Explaining Change through Discursive Institutionalism as the Fourth "New Institutionalism", in: European Political Science Review 2: 1, 1-25.

Schroer, Markus 2006: Räume, Orte, Grenzen. Auf dem Weg zu einer Soziologie des Raums, Frankfurt a. M..

Schroeder, Heike 2008: Analysing Biosafety and Trade through the Lens of Institutional Interplay, in: Young, Oran R./Chambers, W. Bradnee/Kim, Joy A./ten Have, Claudia (Hrsg.): Institutional Interplay: Biosafety and Trade, New York, NY, 49-70.

Schroeder, Heike 2010: Agency in International Climate Negotiations: The Case of Indigenous Peoples and Avoided Deforestation, in: International Environmental Agreements 10: 4, 317-332.

Selin, Henrik/VanDeveer, Stacy D. 2003: Mapping Institutional Linkages in European Air Pollution Politics, in: Global Environmental Governance 3: 3, 14-46.

Soja, Edward 1989: Postmodern Geographies: The Reassertion of Space in Critical Social Theory, London.

Stokke, Olav Schram 2000: Managing Straddling Stocks: The Interplay of Global and Regional Regimes., in: Ocean and Coastal Management 43: 2, $205-234$.

Stokke, Olav Schram 2001: The Interplay of International Regimes: Putting Effectiveness Theory to Work (Fridtjof Nansen Institute Report 14), in: http:// www.fni.no/doc\%26pdf/FNI-R1401.pdf; 5.4.2012. 
Towns, Ann E. 2012: Norms and Social Hierarchies: Understanding International Policy Diffusion "From Below", in: International Organization 66: 2, 179-209.

Victor, David G./Raustiala, Kal/Skolnikoff, Eugene B. 1998: The Implementation and Effectiveness of International Environmental Commitments: Theory and Practice, Cambridge, MA.

Wallbott, Linda 2012: Political in Nature: The Conflict-Fueling Character of International Climate Policies, in: Scheffran, Jürgen/Brzoska, Michael/Brauch, Hans Günter/Link, Peter Michael/Schilling, Janpeter (Hrsg.): Climate Change, Human Security and Violent Conflict: Challenges for Societal Stability, Berlin, 223-241.

Wallbott, Linda 2014a: Goals, Strategies and Success of the African Group in the Negotiations of the Nagoya Protocol, in: Oberthür, Sebastian/Rosendal, G. Kristin (Hrsg.): Global Governance of Genetic Resources. Access and Benefit Sharing after the Nagoya Protocol. New York, 114-131.

Wallbott, Linda 2014b: Indigenous Peoples in UN REDD+ Negotiations: "Importing Power" and Lobbying for Rights through Discursive Interplay Management, in: Ecology and Society 19: 1, 21.

Wallbott, Linda 2014c: 'Keeping Discourses Separate: Explaining the Non-Alignment of Climate Politics and Human Rights Norms by Small Island States in UN Climate Negotiations'. Cambridge Review of International Affairs 27: 4, 736-760.

Wallbott, Linda 2016: 'The Practices of Lobbying for Rights in the Anthropocene Era: Local Communities, Indigenous Peoples and International Climate Negotiations'. In Pattberg, Philipp/Zelli, Fariborz (Hrsg.), Environmental Politics and Governance in the Anthropocene. Institutions and Legitimacy in a Complex World. New York, 213-230.

Wallbott, Linda, Franziska Wolff and Justyna Pozarowska 2014: 'The negotiations of the Nagoya Protocol: Issues, coalitions and process'. In Oberthür, Sebastian und G. Kristin Rosendal (eds), Global Governance of Genetic Resources. Access and Benefit Sharing after the Nagoya Protocol. New York: Routledge, 33-59.

Wallbott, Linda 2019: Negotiating Human Rights in a Changing Climate. The Exploration of Spatiality of Norm Change in Global Politics. Tübingen.

Weidenhaus, Gunter 2015: Soziale Raumzeit, Frankfurt a. M.

Wiener, Antje 2007: Contested Meanings of Norms: A Research Framework, in: Comparative European Politics 5: 1, 1-17.

Wiener, Antje 2008: The Invisible Constitution of Politics: Contested Norms and International Encounters, Cambridge.

Wiener, Antje/Puetter, Uwe 2009: The Quality of Norms is What Actors Make of It: Critical Constructivist Research on Norms, in: Journal of International Law and International Relations 5: 1, 1-16.

Wunderlich, Carmen 2013: Theoretical Approaches in Norm Dynamics, in: Müller, Harald/Wunderlich, Carmen (Hrsg.): Norm Dynamics in Multilateral Arms Control: Interests, Conflicts, and Justice, Athens, GA, 20-50. 
Young, Oran R. 1996: Institutional Linkage in International Society: Polar Perspectives, in: Global Governance 2: 1, 1-24.

Young, Oran R. 1999: The Effectiveness of International Environmental Regimes: Causal Connections and Behavioral Mechanisms, Cambridge, MA.

Young, Oran R. 2002: The Institutional Dimensions of Environmental Change: Fit, Interplay, and Scale, Cambridge, MA.

Zelli, Fariborz 2008: Regime Conflicts in Global Environmental Governance: A Framework for Analysis (Global Governance Working Paper Nr. 36), in: http:// www.glogov.org/images/doc/WP36.pdf; 05.04.2012.

Zelli, Fariborz/Gupta, Aarti/van Asselt, Harro 2012: Horizontal Institutional Interlinkages, in: Biermann, Frank/Pattberg, Philipp (Hrsg.): Global Environmental Governance Reconsidered: New Actors, Mechanisms and Interlinkages, Cambridge, MA, 175-198.

Zimmermann, Lisbeth/von Staden, Andreas/Marciniak, Angela/Wallbott, Linda/Arndt, Friedrich 2013: Muss Ordnung sein? Zum Umgang mit Konflikten zwischen normativen Ordnungen, in: Zeitschrift für Internationale Beziehungen 20: 1, 35-60.

Zürn, Michael/Binder, Martin/Ecker-Erhardt, Matthias/Radtke, Katrin 2007: Politische Ordnung wider Willen, in: Zeitschrift für Internationale Beziehungen 14: 1, 129-164. 